\title{
Unitarity Corrections to the Drell-Yan Process in the Target Rest Frame
}

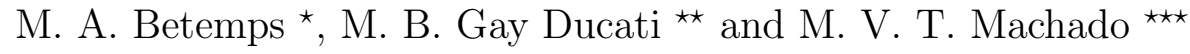 \\ Instituto de Física, Universidade Federal do Rio Grande do Sul \\ Caixa Postal 15051, CEP 91501-970, Porto Alegre, RS, BRAZIL
}

\begin{abstract}
The unitarity corrections effects encoded in the Glauber-Mueller approach are taken into account to calculate the differential cross sections in the DrellYan process in the rest frame. A detailed study of the Drell-Yan process in terms of the $\gamma^{*} q$ transverse separation and the color dipole size, and of the effective dipole cross section, is performed and compared with the available small $x$ data. Estimates for the Drell-Yan cross section at RHIC energies are presented and discussed.
\end{abstract}

13.85.Qk; 12.38.Bx; 12.38.Aw.

Typeset using REVTEX

^E-mail:mandrebe@if.ufrgs.br

${ }^{\star \star}$ E-mail:gay@if.ufrgs.br

${ }^{\star \star \star}$ E-mail:magnus@if.ufrgs.br 


\section{INTRODUCTION}

Both the massive lepton pairs production in hadronic collisions (Drell-Yan) and deep inelastic scattering (DIS) at high energy are the most outstanding processes probing the hadron structure. On the deep inelastic side, a large amount of work has been done to describe the copious data at medium and very small Bjorken scaling variable $x$, based on perturbative QCD [1]. In the high energy domain it has been found that important unitarity corrections should be taken into account regarding the standard pQCD approach [2]. These phenomena are currently denominated perturbative shadowing or saturation effects [3] 6 . Concerning the Drell-Yan sector, the pQCD tools have produced a reasonable theoretical understanding of the main observables, despite the small data set available at present [7]. The forthcoming accelerator experiments (RHIC and LHC) will scan the high energy limit of the hadronic reactions and open a new kinematic window, i.e. smaller $x$ values. In particular, the quark-gluon plasma (QGP), a new state of the hadronic matter predicted by QCD, is expected to be found there [8]. The theoretical description of the QGP production is directly associated to a complete knowledge on saturation effects and the transition region to the high parton densities. In a specific way, since the production scheme for $J / \Psi$ is similar to the Drell-Yan one and the latter does not contain final state effects, DY can be considered as a baseline process to study $J / \Psi$ suppression as a signature of QGP formation [9].

In the fast proton system, the QCD factorization theorem leads to describe the hadronic processes through the convolution of the parton distribution functions (pdf's) with the partonic subprocesses. The latter are completely calculated in pQCD up to higher orders, whereas only the evolution in the factorization scale of the pdf's is determined. Namely, the parton distributions are solutions of the DGLAP evolution equations, whose formalism has been successful in describing both DIS and DY data [1,0]. Recently, an alternative way to study electron-proton and hadronic reactions is claimed by the color dipole picture considering the rest frame description based on $k_{T}$-factorization [3, 10, 11]. Thus, the basic 
blocks are the dipole light-cone wavefunction and the dipole-target cross section. Such an approach has produced an unified way to study the mentioned processes, however its complete connection with the standard DGLAP formalism is not provided yet and deserves further studies.

In the infinite momentum frame, the DY process corresponds to the annihilation of a quark (antiquark) from the projectile with antiquark (quark) of the target into a virtual photon (vector boson), which afterwards decays into a lepton pair [12]. In the leading order (LO) calculation, the DY process has a simple electromagnetic character and it can be promptly given by QED theory. However, the perturbative QCD results at higher orders modify this simple picture. At present, pQCD calculations have been developed up to the second order of the strong coupling constant $\alpha_{s}$ [13]. For practical considerations, in general the involved next orders contributions are taken into account by a phenomenological parameter, namely a $K$ factor which is dependent on the DY kinematic variables.

In the rest frame, the DY process looks like a bremsstrahlung of a virtual photon decaying into a lepton pair, rather than a parton annihilation [11]. The bremsstrahlung of the virtual photon can occur after or before the interaction with the gluonic field of the target. The advantage of this formalism is that the corresponding cross section can be considered in terms of the same dipole cross section extracted from small- $x$ DIS in the color dipole picture [14]. At high energy, the unitarity corrections should be included in the dipole cross section. Such effects have been considered, for example, in the phenomenological model of G.BiernatWusthoff (GBW) [15], which describes DIS and ep diffractive process with good agreement. We notice, however, that the unitarity corrections to the inclusive observables, i.e. total cross section or $F_{2}$, can be hidden into the parametrization based on DGLAP approach, absorbed in the initial conditions, thus providing an excellent data description as seen in the updated NLO QCD fits [16]. More exclusive observables should be useful to clarify this important aspect. After this short remark, we proceed our argumentation. The main disadvantage in GBW is that a dynamical explanation of the saturation phenomenon is lacking. On the other hand, the Glauber-Mueller approach provides a theoretical development concerning 
parton saturation [4, constraining the pQCD description of the dipole cross section. Here, we make use of this formalism to perform a description of the DY process in the rest frame.

The goal of this work is to perform a study of DY at high energies considering the color dipole picture, in a similar way of recent works [17]. Our contribution is based on the use of the dipole cross section calculated in perturbative QCD, through the Glauber-Mueller formula [1], which encodes the unitarity effects (saturation) in the parton densities. This approach takes into account the multiple Pomeron scattering hypothesis in an eikonal way keeping the unitarity of the considered process. A comparison between the phenomenological GBW dipole cross section and the theoretical Glauber-Mueller one is presented, verifying that the two approaches have different behaviors at higher energies. This is due to the dynamical dependence on the gluon distribution in the Glauber-Mueller approach, whose Born term recovers the DGLAP kernel in double log approximation (DLA). The nonperturbative region, i.e. large dipole sizes contributions, is addressed considering the freezing of the gluon distribution under the initial perturbative evolution scale $Q_{0}^{2}$. Then, we present DY calculations in the rest frame of the target at leading order in a $p p$ collision and perform a comparison with the low $x$ DY differential cross section from the E772 Collaboration [18]. We also produce estimates for the cross section at RHIC energies.

The outline of this paper is the following. In the next section we present a brief review of the DY process in the dipole color picture, discussing the range of validity for this approach and showing the role played by the $\gamma^{*} q$ wavefunction. In Sec. (3) we present high parton density effects calculated from the Glauber-Mueller approach, and confront them with the phenomenological GBW model. We estimate the contribution of the saturation effects for the dipole cross section in high energies (LHC and RHIC). In Sec. (4), a parameter-free prediction to the differential DY cross section for the available data at small- $x$ and estimates to RHIC are performed. Finally, in the last section the results are discussed and we present our conclusions. 


\section{DRELL-YAN IN THE COLOR DIPOLE PICTURE}

Before the description of the Drell-Yan process in the rest frame, we would like to review the main kinematical variables and the standard calculations in the laboratory system. This is important to clarify the connection between them and to emphasize the asymmetry projectile-target in the rest frame picture.

In the laboratory system, the lepton pairs are produced in the Drell-Yan reaction where partons from the projectile (fast proton) interact with the proton target [12]. Looking at the parton level, a quark-antiquark pair annihilates into a virtual photon in leading order $q \bar{q} \rightarrow \gamma^{*} \rightarrow l^{+} l^{-}$. The symmetry between target and projectile is very clear, namely we cannot distinguish a quark coming from the proton target or from the incoming beam. The momentum fraction carried by the quark from the projectile is labeled $x_{1}$ and from the target is $x_{2}$. The partonic subprocess above is well known from QED, and the hadroproduction cross section is obtained folding the partonic cross section with the quark (antiquark) densities evaluated at the invariant $M^{2}$, the squared lepton pair mass, chosen here as the factorization scale $\mu_{\mathrm{fac}}^{2}$. Their evolution in $M^{2}$ is given by the standard DGLAP evolution equations. Therefore, the DY differential cross section in leading order is given by

$$
\frac{d^{2} \sigma^{D Y}}{d M^{2} d x_{F}}=\frac{4 \pi \alpha_{\mathrm{em}}^{2}}{9 M^{2} s} \frac{1}{\left(x_{1}+x_{2}\right)} \sum_{f} e_{f}^{2}\left[q_{f}\left(x_{1}, M^{2}\right) \bar{q}_{f}\left(x_{2}, M^{2}\right)+\bar{q}_{f}\left(x_{1}, M^{2}\right) q_{f}\left(x_{2}, M^{2}\right)\right],
$$

where $q[\bar{q}]_{f}\left(x, M^{2}\right)$ are the corresponding quark (antiquark) densities with flavour $f$ and squared charge $e_{f}^{2}$. The center of mass energy squared is $s$ and the usual notation is

$$
\begin{aligned}
x_{F} & =x_{1}-x_{2}, \\
\tau & =x_{1} x_{2}=M^{2} / s .
\end{aligned}
$$

The momentum fractions are rewritten as

$$
\begin{aligned}
& x_{1}=\frac{1}{2}\left(\sqrt{x_{F}^{2}+4 \tau}+x_{F}\right), \\
& x_{2}=\frac{1}{2}\left(\sqrt{x_{F}^{2}+4 \tau}-x_{F}\right),
\end{aligned}
$$


where $x_{F}$ is the longitudinal momentum fraction, labeled Feynman $x$. Indeed, $x_{F}, M$ and $s$ are the kinematic variables experimentally measured, whereas the partonic variables, $x_{1}$ and $x_{2}$, are reconstructed from them.

When we consider the target at rest, the DY process looks like a bremsstrahlung: the quark from the projectile radiates a photon, which carries a fraction $\alpha$ of the light-cone momentum of the initial quark, later decaying into the lepton pair [ see Fig. (1)]. The interaction with the target can occur before or after the photon emission. Thus, although diagrammatically no dipole to be present, the interference among graphs results in a product of two quark amplitudes in the DY cross section, testing the external gluonic field at two different transverse positions [11]. Therefore, a remarkable feature emerging is that the $\gamma^{*} q-N$ interaction can be described by the same dipole cross section as in DIS [14].

In the $\left(\alpha, r_{\perp}\right)$ mixed representation, the photoabsorbtion cross section in deep inelastic is described by the convolution of the wavefunctions, $\Psi_{\gamma^{*}}$, from the virtual photon and the interaction dipole cross section, $\sigma_{q \bar{q}}$. The wavefunctions are considered taking into account the first photon Fock state configuration, namely a $q \bar{q}$ pair. The dipole cross section is modeled phenomenologically based on a matching between the hard and soft pieces, constrained by the DIS available data. The transverse separation of the $q \bar{q}$ pair is $r_{\perp}$, and each quark (or antiquark) of the dipole carries a momentum fraction $\alpha$ (or $1-\alpha$ ), from the incoming photon. The small dipole size configurations can be described through pQCD, whereas the large size ones belong to the nonperturbative domain. Hence, one can write the photoabsorption cross section as a function of the scaling variable $x$ and photon virtuality $Q^{2}$ in the quantum mechanics form [10], 


\section{FIGURES}

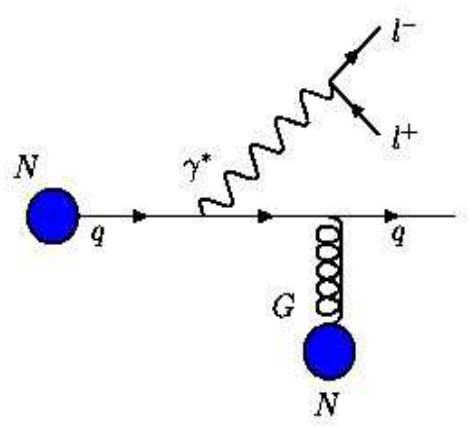

FIG. 1. The Drell-Yan process in the rest frame, depicting one of the possible interactions $\gamma^{*} q$-target (see text).

$$
\sigma_{T, L}\left(\gamma^{*} p \rightarrow q \bar{q}\right)=\int d^{2} r_{\perp} \int_{0}^{1} d \alpha\left|\Psi_{q \bar{q}}^{T, L}\left(\alpha, r_{\perp}\right)\right|^{2} \sigma_{q \bar{q}}\left(x, r_{\perp}\right)
$$

where $T, L$ indicate the transverse and longitudinal contributions to the total cross section. In a similar way, the cross section for radiation of a virtual photon from a quark after scattering on a proton has the following factorized form in the color dipole picture [11]

$$
\frac{d \sigma_{T, L}\left(q p \rightarrow q \gamma^{*} p\right)}{d \ln \alpha}=\int d^{2} r_{\perp}\left|\Psi_{\gamma^{*} q}^{T, L}\left(\alpha, r_{\perp}\right)\right|^{2} \sigma_{q \bar{q}}\left(x_{2}, \alpha r_{\perp}\right)
$$

where we have the same dipole cross section as in DIS. Here $r_{\perp}$ is the photon-quark transverse separation, $\alpha r_{\perp}$ is the $q \bar{q}$ separation and $\alpha$ is the fraction of the light-cone momentum of the initial quark taken away by the photon. We notice the difference with the DIS case, where the dipole separation is just $r_{\perp}$. Here, $\sigma_{q \bar{q}}$ is the cross section for scattering a $q \bar{q}$ pair off a proton which depends on the $q \bar{q}$ transverse separation, and which should take into account the saturation effects at high energy.

The physical interpretation of (17) is similar to DIS in the light-cone approach (LC). The projectile quark state is expanded in its Fock space in the form [11],

$$
|q\rangle=Z_{2}|q\rangle+\Psi_{\gamma^{*} q}^{T, L}\left|q \gamma^{*}\right\rangle+\ldots
$$

where here one has the expansion in terms of the eigenstates from the quark projectile. Instead, in deep inelastic the expansion is constructed from the eigenstates of the incident photon [10]. Here $Z_{2}$ is the renormalization constant. 
The well known LC wavefunctions can be calculated in perturbation theory [10,11], and depend on the transverse separations and momentum fraction $\alpha$. They play an important role in the dilepton mass $M$ dependence. We take the same notation for the LC wavefunctions from [17],

$$
\begin{aligned}
& \left|\Psi_{\gamma^{*} q}^{T}\left(\alpha, r_{\perp}\right)\right|^{2}=\frac{\alpha_{\mathrm{em}}}{\pi^{2}}\left(m_{f}^{2} \alpha^{4} K_{0}^{2}\left(\eta r_{\perp}\right)+\left[1+(1-\alpha)^{2}\right] \eta^{2} K_{1}^{2}\left(\eta r_{\perp}\right)\right), \\
& \left|\Psi_{\gamma^{*} q}^{L}\left(\alpha, r_{\perp}\right)\right|^{2}=\frac{2 \alpha_{\mathrm{em}}}{\pi^{2}} M^{2}(1-\alpha)^{2} K_{0}^{2}\left(\eta r_{\perp}\right) .
\end{aligned}
$$

The functions $K_{0}$ and $K_{1}$ are the modified Bessel functions and the auxiliary variable $\eta$, depending on the quark mass $m_{f}$, is given by

$$
\eta^{2}=(1-\alpha) M^{2}+\alpha^{2} m_{f}^{2}
$$

The hadronic differential cross section for the Drell-Yan process is expressed in a factorized form, embedding the partonic cross section, Eq. (7), into the hadronic environment, in the following way [11],

$$
\frac{d \sigma^{D Y}}{d M^{2} d x_{F}}=\frac{\alpha_{\mathrm{em}}}{6 \pi M^{2}} \frac{x_{1}}{\left(x_{1}+x_{2}\right)} \int_{x_{1}}^{1} \frac{d \alpha}{\alpha^{2}} \sum_{i} e_{i}^{2}\left[q_{i}\left(\frac{x_{1}}{\alpha}\right)+\bar{q}_{i}\left(\frac{x_{1}}{\alpha}\right)\right] \frac{d \sigma\left(q p \rightarrow q \gamma^{*} p\right)}{d \ln \alpha}
$$

where $e_{i}$ is the quark charge. In this frame we use standard kinematical variables $x_{1}=$ $\left(2 P_{2} \cdot q\right) / s$ and $x_{2}=\left(2 P_{1} \cdot q\right) / s$, with $x_{1} x_{2}=\left(M^{2}+q_{T}^{2}\right) / s$, where $P_{1}, P_{2}$ and $q$ are the four momenta of the beam, target and virtual photon, respectively. $M^{2}=q^{2}$ and $q_{T}^{2}$ are the dilepton invariant mass squared and the squared transverse momentum, respectively.

The frame dependence of the space-time interpretation of the DY process can be illustrated by different meanings of $x_{1}$ in different reference frames: we know that in the Breit frame, $x_{1}$ is the momentum fraction of the projectile quark (antiquark) annihilating with the target antiquark (quark). In contrast, evaluating the scalar product referred above in the target rest frame shows that the projectile quark carries momentum fraction $x=x_{1} / \alpha$ (which is larger than $x_{1}$ ), of the parent hadron, and correspondingly, $x_{1}$ is the momentum fraction of the proton carried by the photon. The variable $x_{2}$ is the momentum fraction of the proton carried by the gluon exchange in the $t$-channel. We are benefited with the fact 
that the parton densities $q_{i}$ and $\bar{q}_{i}$ of the projectile enter in the combination $F_{2}^{p}$, which is the structure function of the proton. Therefore, we can rewrite the equation above in the following way,

$$
\frac{d \sigma^{D Y}}{d M^{2} d x_{F}}=\frac{\alpha_{\mathrm{em}}}{6 \pi M^{2}} \frac{1}{\left(x_{1}+x_{2}\right)} \int_{x_{1}}^{1} \frac{d \alpha}{\alpha} F_{2}^{p}\left(\frac{x_{1}}{\alpha}\right) \frac{d \sigma\left(q p \rightarrow q \gamma^{*} p\right)}{d \ln \alpha}
$$

where the summation of the longitudinal and transverse contribution was considered. The factor $\alpha_{\mathrm{em}} /\left(6 \pi M^{2}\right)$ is due to the photon decay into the lepton pair, coming from electrodynamics, the differential cross section $d \sigma\left(q p \rightarrow q \gamma^{*} p\right) / d \ln \alpha$ is taken from equation (7) and our input to $\sigma_{q \bar{q}}$ in this work [4 is given by the standard gluon distribution in the target corrected by saturation effects in the high energy limit. In Eq. (13), the structure of the projectile is described by the $F_{2}^{p}\left(x, Q^{2}\right)$ structure function.

In the rest frame, the process is asymmetric concerning projectile and target, in contrast with the symmetric picture in the Breit frame. The dipole color picture is valid for small $x_{2}$ and it takes into account only the gluonic (sea quarks) sector from the target, disregarding its valence content. However, both valence and sea quarks in the projectile are parametrized in the proton structure function in Eq. ([13) (for a complete discussion see Refs. [17]). Although at present there is little range of experimental measurements in the kinematical limit of validity of the color dipole approach, it should provide reasonable results when one considers smaller $x_{2}$ than the currently available. The high energy accelerators LHC and RHIC will open a wider kinematical window towards smaller $x_{2}$ values allowing to test rest frame calculations properly.

To conclude this section, we analyse the behavior of the wavefunctions in the relevant kinematic variables. As it will be shown, they play the role of a weight to the dipole cross section concerning the transverse separations. In Eqs. (6-7), large $r_{\perp}$ configurations are suppressed in the integrated cross section, controlling the nonperturbative contributions (large transverse distances domain) to the observables. In the deep inelastic case, the LC wavefunctions dependence on the radius $r_{\perp}$ at fixed photon virtuality $Q^{2}$ is discussed in Refs. [19]. For the Drell-Yan case, the weight functions are given by: 


$$
W_{\gamma_{*} q}^{T, L}\left(r_{\perp}, M^{2}\right)=r_{\perp} \int \frac{d \alpha}{\alpha} F_{2}^{p}\left(x_{1} / \alpha, M^{2}\right)\left|\Psi_{\gamma^{*} q}^{T, L}\left(\alpha, r_{\perp}\right)\right|^{2}
$$

In Fig. (2) one shows separately the longitudinal and transverse results for $W^{T, L}\left(r_{\perp}, M^{2}\right)$ as a function of the photon-quark transverse separation $r_{\perp}$ at fixed lepton pair mass $M$. The choosen momentum fraction was $x_{1} \approx x_{F}=0.525$, since it is a typical experimental value (see Sec. 4). Considering this $x_{1}$ value, the proton structure function is insensitive to the lepton pair mass range because it is in the scaling region. Regarding the quark mass, here one takes an effective light quark mass $m_{f}=0.2 \mathrm{GeV}$ in the wavefunctions.

For the transverse contribution, meaning the upper plot in Fig. (2), one verifies that the weight function selects from small up to intermediate photon-quark sizes. This means that it is selecting small dipole sizes $\left(\alpha r_{\perp}\right)$ in a similar way to deep inelastic scattering, since $x_{1} \leq \alpha \leq 1$. For our purpose here, the $x_{1}$ values reside close to $x_{F}$, then the conclusions in the following should hold when the weight factor is applied to the dipole cross section depending on $\alpha r_{\perp}$. A steep increasing as $r_{\perp} \rightarrow 0$ comes from the behavior of the function $K_{1}\left(\eta r_{\perp}\right) \sim 1 /\left(\eta r_{\perp}\right)$ at this limit. Concerning the dependence on $M$, as the invariant mass increases the contribution looks smaller.

Regarding the longitudinal contribution, the lower plot in Fig. (2), the weight function selects smaller dipole sizes (and $\gamma^{*} q$ transverse sizes) in comparison with the transverse contribution. Moreover, the function is narrower as $M$ increases, meaning that larger invariant mass scans smaller $r_{\perp}$. A well known fact is that the longitudinal contribution is higher twist, i.e. it is suppressed by a power of $1 / M^{2}$ when compared with the transverse one [10]. This feature actually remains in the Drell-Yan case. Moreover, the peaks appearing in the plot are due to the balancing between the asymptotic behavior at $r_{\perp} \rightarrow 0$ of the function $K_{0}\left(\eta r_{\perp}\right) \sim-\log \left(\eta r_{\perp}\right)$ and the linear $r_{\perp}$ factor in Eq. (14). 


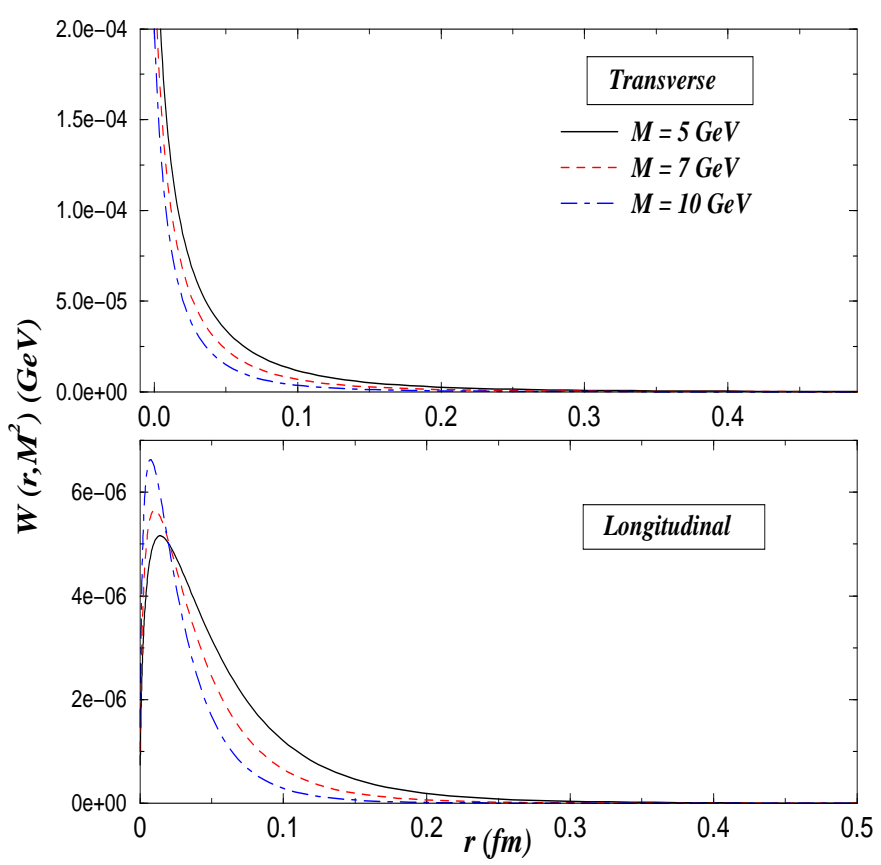

FIG. 2. The longitudinal and transverse contributions for $W\left(r_{\perp}, M^{2}\right)$ as a function of the $\gamma^{*} q$ transverse size $r_{\perp}$ at fixed lepton pair mass $M$, for $x_{1} \approx x_{F}=0.525$.

Having addressed the main features of the color dipole framework, namely kinematic definitions and the description of Drell-Yan process in the rest frame, in the next section one introduces our model for the dipole cross section satisfying unitarity requirements.

\section{THE GLAUBER-MUELLER APPROACH}

The cross section for a color dipole-nucleon scattering is a well known quantity, which was first proposed in the BFKL framework [20]. The dipole interacts with the target through a perturbative Pomeron, described in terms of the ladder diagrams. From the $k_{T}$-factorization framework [21], the scattering process can be written as the convolution of the projectile impact factor and the unintegrated gluon structure function from the target, whose dynamics is determined by the evolution kernel. The possible orderings in the transverse momentum $k_{T}$ in these graphs produce the DGLAP or the BFKL dynamical evolutions. In particular, considering small $r_{\perp}$ configurations from the dipole and the $k_{T}$-factorization one obtains, 


$$
\sigma_{q \bar{q}}\left(x, r_{\perp}\right)=\frac{\pi^{2} \alpha_{s}}{3} r_{\perp}^{2} x G_{N}^{D G L A P}\left(x, \frac{4}{r_{\perp}^{2}}\right)
$$

where $x G_{N}^{D G L A P}\left(x, \tilde{Q}^{2}\right)$ is the standard DGLAP gluon distribution at momentum fraction $x$ and virtuality scale $\tilde{Q}^{2}=4 / r_{\perp}^{2}$. An extensive phenomenology has been made using the result above for the inclusive structure function and the vector meson production [19]. In particular, we call attention to the specific value of the scale $r_{0}^{2}$ appearing in the virtuality scale $\tilde{Q}^{2}=r_{0}^{2} / r_{\perp}^{2}$. We use the $r_{0}^{2}=4$ throughout this paper, however other values are equivalent at leading logarithmic level [19].

A well defined feature from the data on $F_{2}$ and on the gluon distribution at high energies, i.e. smaller $x$, is that they present a steep increasing as $x$ decreases. Indeed, experimentally $F_{2} \sim x G \sim x^{-\lambda}$, where the exponent ranges from 0.08 (Regge phenomenology) up to 0.5 (LO BFKL calculations). Such a behavior extrapolated to asymptotic energies violates unitarity requirements and a control should be considered. The scale where these effects start to be important is associated to a region between hard and soft dynamics [22] (pQCD versus Regge) or belonging to the high density QCD domain [4]. Here, we are interested in the last case (for a recent review, see [23]). In QCD, the taming of the gluon distribution at high energies is taken into account through multiple Pomeron scattering encoded in the eikonallike frameworks [24]. Such a procedure provides the unitarization of the Born Pomeron cross section leading to a softer growth with energy. Indeed, the asymptotic calculations have produced an unified $\ln (1 / x)$ pattern for the cross section and gluon function instead of a truly saturated one 25].

In this work we use Eq.(15), where the standard DGLAP gluon distribution is replaced by the modified one. In the following we shortly review how the unitarity corrections are implemented through the Glauber-Mueller approach [4]. The starting point for the derivation is the interaction of a virtual probe particle, in our case a virtual gluon, with the nucleon. In the space-time picture of this process, the virtual probe decays in a gluon-gluon $(G G)$ pair having transverse separation $r_{\perp}$. In the high energy limit, $r_{\perp}$ is considered frozen during the interaction for $x<<1 /\left(2 m_{N} R_{N}\right)$, where the nucleon $N$ has mass $m_{N}$ and geometric 
transverse size $R_{N}$.

The absorption cross section of a virtual gluon $\left(G^{*}\right)$ with virtuality $Q^{2}$ and Bjorken $x$ can be written in the form,

$$
\sigma^{G^{*}}\left(x, Q^{2}\right)=\int_{0}^{1} d z \int \frac{d^{2} r_{\perp}}{\pi} \int \frac{d^{2} b}{\pi}\left|\Psi^{G^{*}}\left(Q^{2}, r_{\perp}, x, z\right)\right|^{2} \sigma_{N}^{G G}\left(x, r_{\perp}\right)
$$

where $z$ is the fraction of the energy carried by the gluon, $b$ is the impact parameter variable and $\Psi^{G^{*}}$ is the wavefunction for the transversally polarized gluon generating the pair. The cross section of the interaction $G G$ pair with the nucleon $\sigma_{N}^{G G}$ depends on energy $x$ and transverse separation $r_{\perp}$. This description is valid in leading $\ln (1 / x)$ approximation, however in the double log approximation (DLA) of perturbative QCD one obtains [4],

$$
\sigma_{N}^{G G}\left(x, r_{\perp}\right)=\frac{3 \pi^{2} \alpha_{s}}{4} r_{\perp}^{2} x G^{D G L A P}\left(x, \frac{4}{r_{\perp}^{2}}\right)
$$

The unitarity constraint to the cross section above is expressed by the eikonal-like Glauber (Mueller) formula, hence the the gluon structure function can be written as [23],

$$
x G\left(x, Q^{2}\right)=\frac{4}{\pi^{2}} \int_{x}^{1} \frac{d x^{\prime}}{x^{\prime}} \int_{\frac{4}{Q^{2}}}^{\infty} \frac{d r_{\perp}^{2}}{\pi r_{\perp}^{4}} \int \frac{d^{2} b}{\pi} 2\left\{1-e^{-\frac{1}{2} \sigma_{N}^{G G}\left(x^{\prime}, r_{\perp}^{2} / 4\right) S(b)}\right\} .
$$

The explicit integration limits for the $z$ (rewritten through the variable $x^{\prime}$ ) and transverse separation come from the physical kinematic range allowed in the process (for detailed discussions, see [4]). The impact parameter $b$ dependence, is parametrized in the profile function $S(b)$. It contains information about the angular distribution of the scattering in the nucleon case and the nucleon distribution inside the nucleus in the nuclear case.

The Born term, in the expansion of Eq. (18) respect to $\sigma_{N}^{G G}$, provides the DGLAP evolution in double logarithmic approximation (DLA). The remaining terms in the series contribute to the saturation effects to the Born term. For simple calculations, the profile function $S(b)$ is parametrized as a Gaussian distribution, $S(b)=\frac{1}{\pi R_{A}^{2}} e^{-\frac{b^{2}}{R_{A}^{2}}}$, where $R_{A}$ is the target size, which is a free parameter to be determined from data. Then, putting all together and performing the integration over impact parameter $b$ in Eq. (18), one obtains

$$
x G\left(x, Q^{2}\right)=\frac{2 R_{A}^{2}}{\pi^{2}} \int_{x}^{1} \frac{d x^{\prime}}{x^{\prime}} \int_{1 / Q^{2}}^{1 / Q_{0}^{2}} \frac{d r_{\perp}^{2}}{r_{\perp}^{4}}\left(\gamma_{E}+\ln \left[\kappa_{G}\left(x^{\prime}, r_{\perp}^{2}\right)\right]+E_{1}\left[\kappa_{G}\left(x^{\prime}, r_{\perp}^{2}\right)\right]\right),
$$


where $\gamma_{E}$ and $E_{1}(x)$ are the Euler constant and the exponential integral, respectively. The packing factor $\kappa_{G}=\left(3 \pi \alpha_{s} r_{\perp}^{2} / 2 R_{A}^{2}\right) x G^{\text {DGLAP }}$, sets the scale where saturation effects are starting. Namely, the saturation scale $Q_{s}^{2}$ is defined through the expression $\kappa_{G}\left(x, Q_{s}^{2}\right)=1$.

Since the Glauber-Mueller approach is valid in DLA, for practical reasons in Refs. [4, [5] a procedure was introduced to extend the formalism to the full experimental kinematic range available. The final result contains the full DGLAP kernel corrected by contributions calculated in DLA,

$$
\begin{aligned}
x G_{N}^{G M}\left(x, Q^{2}\right) & =x G\left(x, Q^{2}\right)[E q \cdot(18)] \\
+ & x G^{D G L A P}\left(x, Q^{2}\right)-\frac{\alpha_{s} N_{c}}{\pi} \int_{x}^{1} \frac{d x^{\prime}}{x^{\prime}} \int_{Q_{0}^{2}}^{Q^{2}} \frac{d Q^{\prime 2}}{Q^{\prime 2}} x^{\prime} G^{D G L A P}\left(x^{\prime}, Q^{2}\right),
\end{aligned}
$$

where this modification is necessary to obtain a realistic approach in the region of not very small- $x$. The above equation includes $x G^{D G L A P}\left(x, Q^{2}\right)$ as the initial condition for the gluon distribution and gives $x G^{D G L A P}\left(x, Q^{2}\right)$ as the first term of the expansion with respect to $\kappa_{G}$. One needs to subtract the Born term of Eq. (18) in order to avoid double counting, which is the meaning the last term in equation above.

From the discussions and definitions above, we should use as dipole cross section in our further calculations the following expression,

$$
\sigma_{q \bar{q}}^{G M}\left(x, r_{\perp}\right)=\frac{\pi^{2} \alpha_{s}}{3} r^{2} x G_{N}^{G M}\left(x, \frac{4}{r_{\perp}^{2}}\right) .
$$

The resulting corrected gluon distribution (Eq. (20)) has been applied for a comprehensive phenomenology in DIS process, considering the formulae above as the gluon input for the observables calculated in the Breit frame (structure functions, $F_{2}$ slope, etc.). Recently, the GM dipole cross section has been applied in calculations of the DIS structure functions in the dipole color picture (see, Ref. [26]). For instance, the structure functions description, in particular the latest $F_{2}$ data, can be seen at figures (3) and (5) of the Ref. [26], using that formalism. 


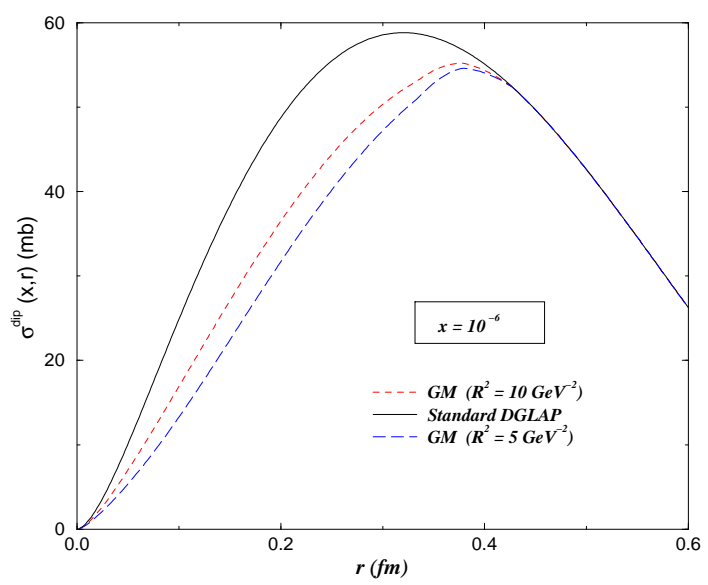

FIG. 3. The color dipole cross section as a function of the dipole size $r=\alpha r_{\perp}$ at fixed $x_{2}=10^{-6}$. The solid line corresponds to the result without unitarity corrections; the dotted line is the GM prediction using $R^{2}=10 \mathrm{GeV}^{-2}$, and the dashed one is for $R^{2}=5 \mathrm{GeV}^{-2}$.

Now, one discusses in a detailed way the main characteristics emerging from the GlauberMueller dipole cross section. To do this, in Fig. (3) one shows the Glauber-Mueller dipole cross section as a function of dipole transverse size $r=\alpha r_{\perp}$ at fixed momentum fraction $x_{2}$. For sake of a better illustration on the partonic saturation effects, one takes a very small value for $x=10^{-6}$. Hereafter, one is using the GRV gluon distribution at leading order [27]. Here we use the GRV94 parametrization, since it has been considered as a robust in a comprehensive phenomenology concerning unitarity corrections [4,5, 26, 28, 30], which includes a related $R$ determination, intrinsic to the Glauber-Mueller approach [28]. The use of the others pdf's [16], implies in a determination of the corresponding value of the parameter $R$ as well as the enhancement of the already present uncertainty about the nonperturbative contribution. For sake of illustration, in order to test the sensitivity to the choice of pdf's set [16], in the Ref. [19] such a study has been performed and it has been found that the deviations among the parametrizations are more important in the high virtuality region (very small $r$ ). However, in this region the color transparency behavior $\sim r^{2}$ dominates, absorbing possible sensibility in the specific selected parametrization.

The solid line corresponds to the standard DGLAP calculation (without saturation), Eq. (15), whereas the remaining ones result from unitarity corrections for two different target 
sizes. The general shape in terms of the dipole size comes from the balancing between the color transparency $\sigma \sim r_{\perp}^{2}$ behavior and the gluon distribution shape. These features are depicted in the plots in Fig. (四), where one verifies a visible scaling of $x G\left(x, \tilde{Q}^{2}\right)$ versus $\tilde{Q}^{2}$ (left plot) and its dependence on $r=\alpha r_{\perp}$ (right plot).

The difference in the strength of the unitarity corrections associated with the target size is a well known fact. From the data analysis, its value ranges from $R^{2}=5-10 \mathrm{GeV}^{-2}$, where smaller radius produces strongest corrections [ see Fig. (3)]. For our calculations we choose the low value $R^{2}=5 \mathrm{GeV}^{-2}$, corroborated by studies in the inclusive structure function and its derivative [28].
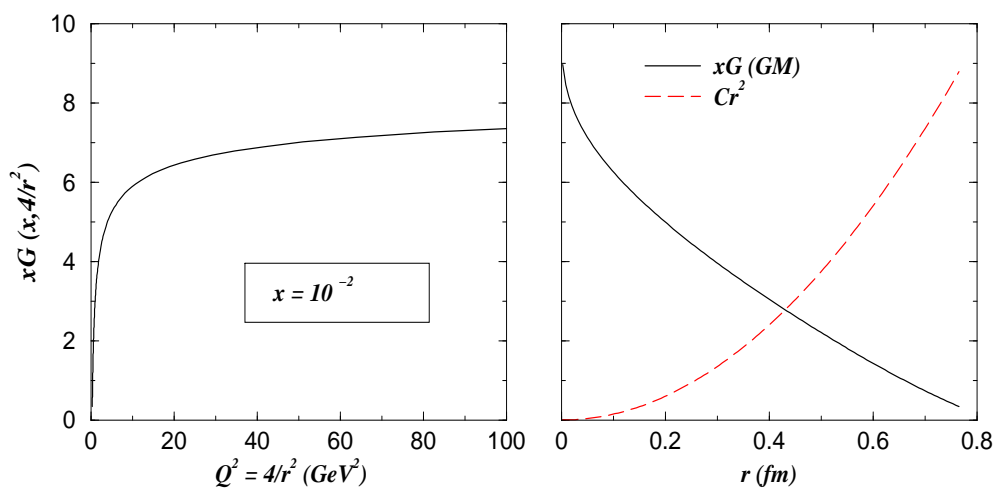

FIG. 4. The plot on the left shows the GM gluon distribution (GRV parametrization input) as a function of the scale $\tilde{Q}^{2}=4 / r^{2}$ at fixed $x=10^{-2}$. On the right, GM gluon distribution versus $r$ and the color transparency behavior $\sigma_{\text {dip }} \sim C r^{2}$ (for illustration one uses a free normalization $C=15)$

For sake of comparison, one considers the phenomenological model of Ref. [15] (GBW), which has produced a good description of HERA data in both inclusive and diffractive processes. It is constructed interpolating the color transparency behavior $\sigma_{\text {dip }} \sim r_{\perp}^{2}$ for small dipole sizes and a flat (saturated) behavior for large dipole sizes $\sigma_{d i p} \sim \sigma_{0}$ (confinement). The expression has the eikonal-like form,

$$
\sigma_{q \bar{q}}(x, r)=\sigma_{0}\left[1-\exp \left(\frac{r^{2} Q_{0}^{2}}{4\left(x / x_{0}\right)^{\lambda}}\right)\right],
$$

where $Q_{0}^{2}=1 \mathrm{GeV}^{2}$ and the three fitted parameters are $\sigma_{0}=23.03 \mathrm{mb}, x_{0}=3.0410^{-4}$ and $\lambda=0.288$ and $R_{0}(x)=\left(x / x_{0}\right)^{\lambda / 2}$ is the saturation radius. In GBW, saturation is 
characterized by the $x$-dependent saturation radius $Q_{s}^{2}(x)=1 / R_{0}^{2}(x)$ instead of the scale coming from Glauber-Mueller, $\kappa_{G}\left(x, Q_{s}^{2}\right)=1$, which can be easily extended for the nuclear case [4].

Although GBW and GM are distinct approaches, the small $x$ DIS data are equally well described by both models. In particular the structure functions have been systematically described using the GM formalism, see for instance Refs. [5, 26, 29, 30]. The main advantageous feature of GM in relation to the GBW is the dipole cross section providing a deep connection with the gluon distribution, the leading quantity at high energies. Concerning GBW, we point out the following shortcomings and disadvantages in comparison with GM approach: $(a)$ it is strictly a parametrization available for the small $x$ HERA data; $(b)$ there is no direct connection with the gluon content; $(c)$ it does not match DGLAP evolution equations; $(d)$ it does not consider the impact parameter dependence of the process; $(e)$ it leads to a quite strong saturation scenario in contrast with the other available approaches; $(f)$ concerning the hadron-hadron collisions, using GBW to calculate the pion-proton total cross section (convoluting the dipole cross section with the pion wavefunction) it predicts non-realistic results, i.e. the cross section saturates at $\sim 23.03 \mathrm{mb}$ in high energies.

In Fig. (5) one shows the Glauber-Mueller dipole cross section as a function of the dipole size $r=\alpha r_{\perp}$ for two typical $x_{2}$ values. In the lower plot, for $x_{2}=10^{-2}$, the GM cross section underestimates the GBW one. However, as $x_{2}$ decreases the gluon distribution in the proton rises and the dipole cross section increases. This feature is depicted in the upper plot, for a small $x_{2}=10^{-6}$, where GM overestimates GBW by a factor two at intermediate $r \sim 0.3$. An immediate consequence from the plots is that our prediction lies lower than GBW at $x_{2} \approx 10^{-2}$ and higher for smaller $x_{2}$. We discuss these features in a theoretical and phenomenological point of view when performing the comparison with available data in the next section. 


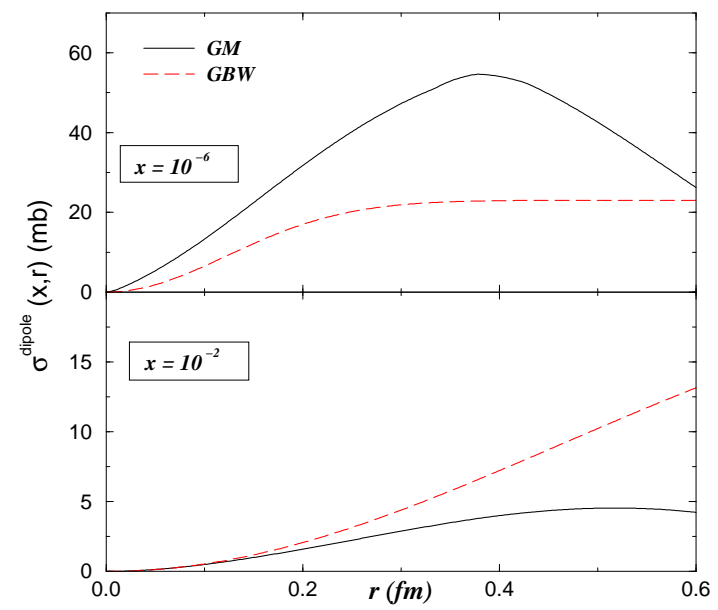

FIG. 5. The GM dipole cross section as a function of the dipole size $r=\alpha r_{\perp}$ at two typical $x_{2}$ values. The GBW result is also shown for sake of comparison.

\section{RESULTS AND DISCUSSIONS}

This section is devoted to perform theoretical predictions for the available data on DY process and the forthcoming ones from RHIC or LHC. In the previous section, we presented a parameter-free Glauber-Mueller dipole cross section which matches leading log gluon evolution and contains corrections from unitarity effects (parton saturation) at higher energies. Therefore, this provides a clear dynamical description of the observables depending on the gluonic content of the target, also when it is a nuclear one.

Although perturbative QCD provides reliable results at small distances (small dipole sizes), the nonperturbative sector is still far from being completely understood. The usual pdf's are evolved from a perturbative initial scale $Q_{0}^{2}=M_{0}^{2} \approx 1 \mathrm{GeV}^{2}$, and there is little information about the behavior at $Q^{2} \leq Q_{0}^{2}$, where the perturbative description is not even justifiable. In general one makes use of Regge phenomenology to estimate those contributions (see, for instance [19]), and extrapolating to lower virtuality regions (large dipole sizes) one needs an ansatz regarding the nonperturbative sector.

The use of the GRV94 parametrization [27] in our calculations, bearing in mind that $Q^{2}=$ $4 / r^{2}$, meaning its evolution initial scale is $Q_{0}^{2}=0.4 \mathrm{GeV}^{2}$, allows to scan dipole sizes up to $r_{\text {cut }}=\frac{2}{Q_{0}} \mathrm{GeV}^{-1}(=0.62 \mathrm{fm})$. The cut off $\left.r_{(\text {cut }}\right)$ defines the transverse distance scale matching 
the perturbative and nonperturbative sector. For the most recent parametrizations, where $Q_{0}^{2} \sim 1 \mathrm{GeV}^{2}\left(r_{\text {cut }} \approx 0.4 \mathrm{fm}\right)$ the amount of nonperturbative contribution in the calculations should increase. An additional advantage is that GRV94 does not include non-linear effects to the DGLAP evolution since the parametrization was obtained from rather large $x$ values. This feature ensures that the parametrization does not include sensible unitarity corrections (perturbative shadowing) in the initial scale.

Now, we should introduce an ansatz for the large transverse separation region. A more phenomenological way is to match the pQCD dipole cross section with the typical hadronic one $\sigma_{\pi N}$ at $r_{\text {cut }}$, for instance as performed in 19 . Nevertheless, due to the large growth of the pQCD dipole cross section at high energies and to take a more simple technical procedure we choose an alternative way: the gluon distribution is frozen at scale $r_{\text {cut }}$, namely $x G\left(x, \tilde{Q}_{\text {cut }}^{2}\right)$. Then, the large distance contribution $r \geq r_{\text {cut }}$ reads as,

$$
\sigma_{q \bar{q}}^{G M}\left(x, r \geq r_{\text {cut }}\right)=\frac{\pi^{2} \alpha_{s}}{3} r_{\text {cut }}^{2} x G_{N}^{G M}\left(x, \frac{4}{r_{\text {cut }}^{2}}\right)
$$

In a more rigorous analyses, one should substitute the freezening scale $\tilde{Q}_{\text {cut }}^{2}$ for the saturation scale $Q_{s}^{2}(x)$ to take into account a realistic value of the gluon anomalous dimension in all kinematic regions.

Concerning our ansatz for the large $r$ region, one verifies in Ref. [26] that it produces reasonable data description, mainly the normalization of the structure functions. An improvement for the non-perturbative contribution is performed there, where a cut off in the $r$ integration $\left(0 \leq r \leq r_{c u t}\right)$ and the addition of a soft Pomeron term are considered. However, if we do not introduce this improvement, the normalization of the structure functions remains unaffected. For completeness, the consideration of a soft term for the non-perturbative contribution was also taken into account in Ref. [19]. However, for the same reasons above, we choose the technically more simple procedure. 


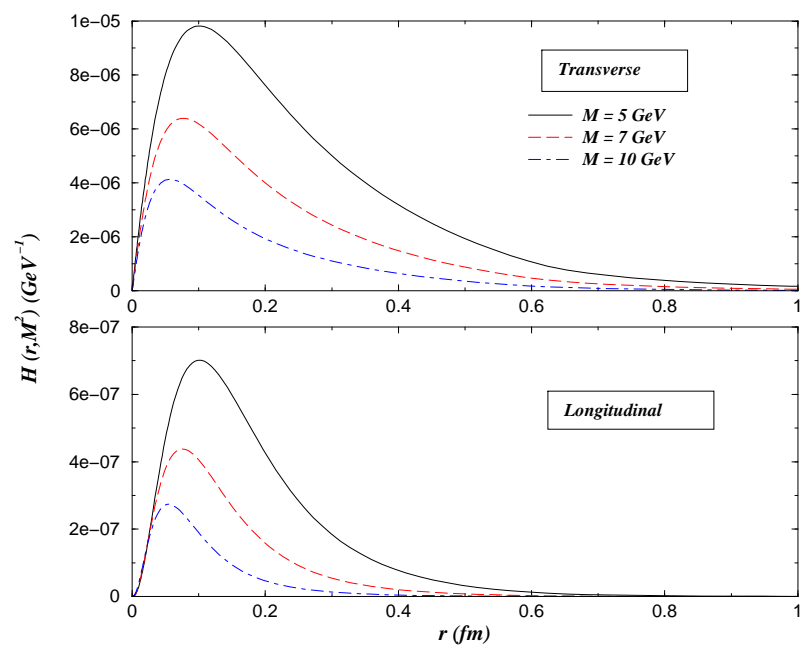

FIG. 6. The profile $H_{T, L}\left(r_{\perp}, M^{2}\right)$ as a function of the $\gamma^{*} q$ transverse separation $r_{\perp}$ at typical mass $M$ values. One uses $x_{2}=10^{-2}$ and GRV parametrization input.

To illustrate the role played by the small and large transverse separations in the description of the observables, in Fig. (6) one shows the profile of the $r_{\perp}$-integration from Eq. (13) as a function of the $\gamma^{*} q$ transverse separation $r_{\perp}$ at typical mass $M$ values. It is labeled here as $H_{T, L}\left(r_{\perp}, M^{2}\right)$. The momentum transfer is fixed at $x_{2}=10^{-2}$, since the low $x_{2}$ data available lie at this magnitude. For the proton structure function $F_{2}^{p}\left(x_{1} / \alpha, M^{2}\right)$, describing the quark content of the projectile, we use the ALLM updated parametrization [31] (good agreement with HERA data at large $x$ ). Both transverse and longitudinal profiles are presented.

The main contribution for the profiles comes from the asymmetric peaks which are shifted to larger $r_{\perp}$ as $M$ diminishes. For instance, in the transverse profile, the peak lies at $r_{\perp} \approx 0.06 \mathrm{fm}$ for $M=10 \mathrm{GeV}$ whereas at $M=5 \mathrm{GeV}$ it takes values $r_{\perp} \approx 0.1 \mathrm{fm}$. As it was verified in Sec. (2), the longitudinal sector selects smaller transverse sizes $r_{\perp}$ than the transverse one. Indeed, from the upper plot, non-zero contributions are obtained up to large $r_{\perp} \approx 1 \mathrm{fm}$. The higher twist character of the longitudinal piece is verified through the magnitude scale of $H_{L}\left(r_{\perp}, M^{2}\right)$. Due to the fact that the nonperturbative sector dominates for $r_{\text {cut }}=\left(\alpha r_{\perp}\right)_{\text {cut }} \geq 0.62 \mathrm{fm}$ using the GRV input, a significant part of the contributions comes from the soft region where $\alpha$ is small (soft quark). This is in agreement with the 
expectations that important soft contributions take place in Drell-Yan process (see related discussions at 35).

Now, we are able to compare the results with the available data. Since the color dipole picture is valid at small momentum fraction $x_{2}$, one needs to select the experimental data covering this requirement. The lowest $x_{2}$ data were obtained in the fixed-target dimuon production at E772 Collaboration [18], where we select the points with $x_{2}<0.1$ following the similar procedure of [17]. In Fig. (7), one presents the calculation Eq. (13) using the Glauber-Mueller dipole cross section (the solid line) at fixed $x_{F}$ and center of mass energy $\sqrt{s}=38.8 \mathrm{GeV}\left(0.03 \leq x_{2} \leq 0.09\right)$. It should be stressed that this kinematical region scans the validity limit of the color dipole picture. The curves underestimate similar calculations in Ref. [17], which uses the phenomenological GBW dipole cross section. Such a result is actually expected from our conclusions in the previous section where GM underestimate GBW at $x_{2}=10^{-2}$ [see lower plot in Fig. (5)].

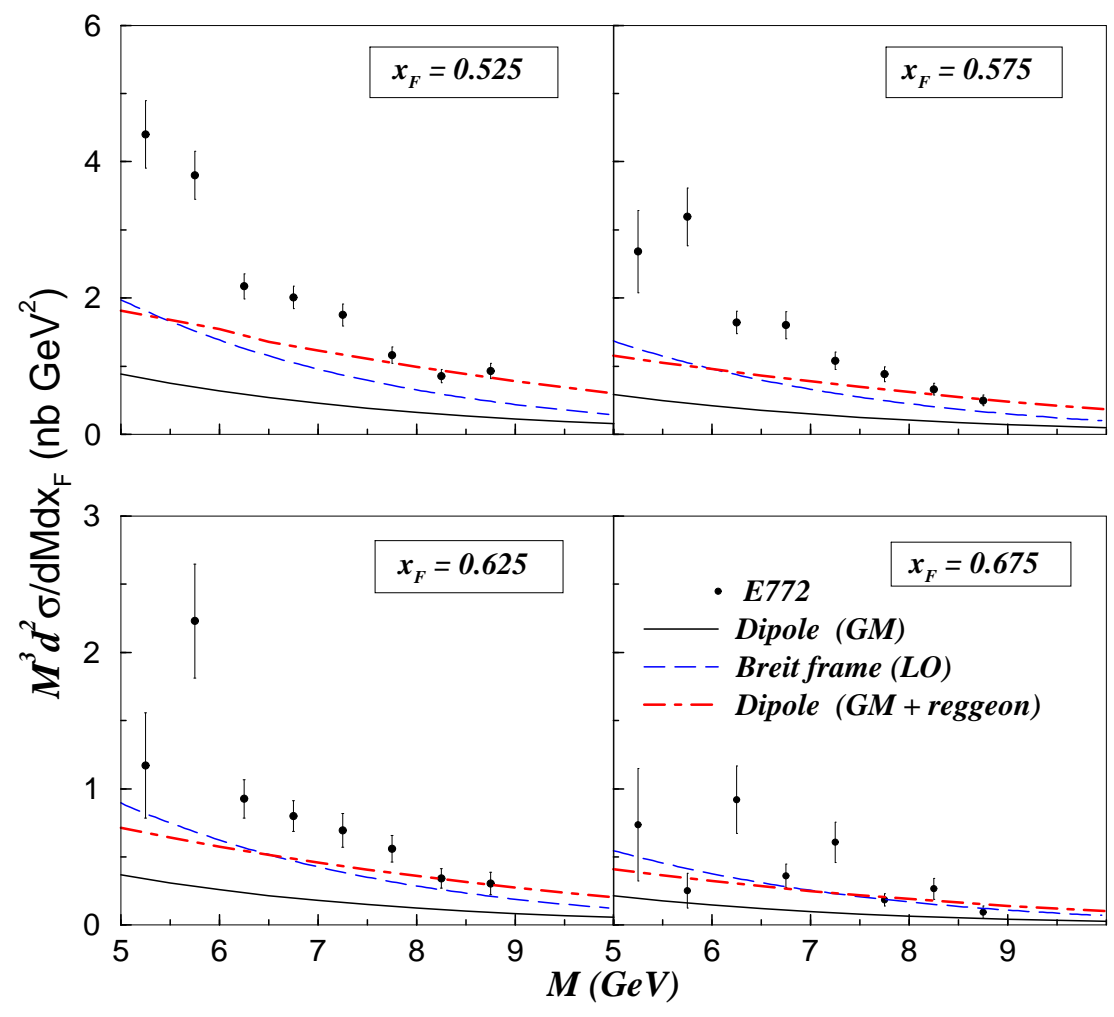


FIG. 7. The DY differential cross section per nucleon versus $M$ for the available small $x_{2}$ data [18] $(\sqrt{s}=38.8 \mathrm{GeV})$ at fixed $x_{F}$ in $p D$ reaction. The solid line corresponds to the Glauber-Mueller dipole cross section. The dashed one is the LO Breit system calculation and the dot-dashed line corresponds to the Glauber-Mueller added of a reggeon contribution.

The experimental data analysed above are for $x>10^{-2}$. Therefore, the Eq. (21) is known to have corrections at larger $x$ values due to the exchange of quarks rather than gluons, in the $t$-channel, corresponding to a reggeon instead of a Pomeron exchange [32]. The secondary reggeon contribution corresponds to an amplitude with quark-antiquark pair t-channel exchange. The leading double-logarithmic asymptotics of such an amplitude was calculated in perturbative QCD in Ref. [33]. The quark-antiquark cut occurs in the $j$-plane at $\omega_{0}(t)=\sqrt{2 C_{F} \alpha_{s} / \pi}$, where $C_{F}=\left(N_{c}^{2}-1\right) / 2 N_{c}$ and $\alpha_{s}$ is the strong coupling constant. That value is very close to the phenomenological intercepts of the $\omega, \rho$ trajectories, i.e. $\alpha_{R}(0) \simeq 0.5$. Our expression for the dipole cross section, Eq. (21), considers only sea contribution for the process (gluon radiation), being equivalent in the Regge terminology to the hard Pomeron. The correspondent valence-like, which corresponds to the reggeon contribution, is lacking in our analysis above. In order to simulate the valence content in the calculations, we parametrize that piece in the following form [34]:

$$
\sigma_{I R}(x, r)=N_{I R} r^{2} x^{0.4525}(1-x)^{3},
$$

where we have used the reggeon intercept $\alpha_{I R}(0)=0.5475$ and the threshold factor for the large $x$ region [34]. To reproduce similar results as presented in Ref. [17], one considers the constant value $N_{I R}=8$ (to obtain a $\sigma_{I R}$ in $\mathrm{GeV}^{-2}$ ). The $r^{2}$ factor ensures the correct scaling.

In the plots (Fig. 7) one shows also the LO Breit system calculation, Eq. (1), which is the dashed line. The color dipole result considering only the gluon content (sea quarks), Eq. (21), lies below LO fast proton frame one at $x_{2} \approx 10^{-2}$ (where the presented data are available). However, in this kinematical region the valence quark content competes with the sea one and such a difference should be expected. Now, we introduce the valence content, 
parametrized in Eq. (24) and added to the Eq. (21): as a result our data description has been improved, equivalently to the calculations in [17] considering the GBW model. As $x_{2}$ decreases the gluonic content of the target drives the observables and the color dipole considering only Eq. (21) should produce quite reliable results. We have verified this feature and have found that the reggeon contribution for the RHIC energies is completely negligible.

Here, some comments about higher order corrections are timely. The color dipole approach results for total (virtual) photon cross section are equivalent to those ones obtained by $k_{T}$-factorization [21] in the leading logarithmic approximation. However, the inclusion of higher order effects in the $k_{T}$-factorization approach turns the equivalence incompatible: the conservation of the transverse positions and sizes of the colliding objects is violated [36]. Therefore, the introduction of higher orders contributions into the dipole cross section must be taken with some care. Moreover, deep inelastic and Drell-Yan have a quite different scenario concerning NLO and NNLO corrections. In DIS, calculations considering up to NNLO resummations have been performed and it was found that they are small [37. Instead, in Drell-Yan even the NLO calculations produce corrections up to a factor of two, diminishing as the energy increases [7, 13]. Keeping in mind the discussion above, at the moment we are unable to perform in the dipole color picture an equivalent NLO (Breit frame) calculation, since at the present the wavefunctions are not still available at NLO accuracy.

In order to address the color dipole picture at high energies, the DY differential cross section for RHIC energies, $\sqrt{s}=500 \mathrm{GeV}$, is shown in Fig. (8) for the same fixed $x_{F}$. There, the $x_{2}$ reaches values of order $10^{-4}$ and unitarity effects are important. The solid lines are the Glauber-Mueller estimates, Eq. (21), and the dot-dashed ones are the rest frame calculations with DGLAP gluon distribution, Eq. (15). The curves overestimate similar calculations in Ref. [17], which uses the phenomenological GBW dipole cross section. Such a behavior is expected from our previous conclusions where GM overestimates GBW at smaller $x_{2}$ due to the growth of the gluon distribution at higher energies [see the upper plot in Fig. (5)]. Concerning the rest frame non-corrected DGLAP input, the Glauber-Mueller underestimates them due to the significant corrections coming from unitarity effects (parton saturation). 
Moreover, we have obtained a result almost similar to the LO Breit frame calculations at the RHIC energies, suggesting a good consistency in both frameworks. From the plots one verifies that the deviations are more significant as $M$ diminishes, corresponding to smaller $x_{2}$ values. In absolute values, the corrections at RHIC energies reach up to $\approx 20 \%$.

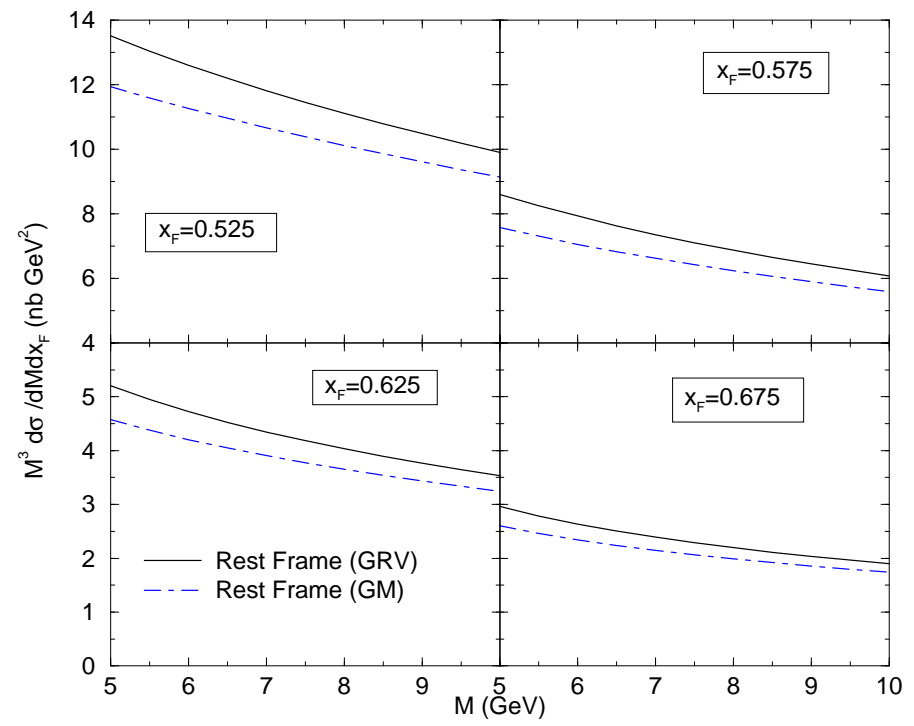

FIG. 8. The DY differential cross section per nucleon versus $M$ for the RHIC energies $(\sqrt{s}=500$ $\mathrm{GeV}$ ) at fixed $x_{F}$ in $p d$ reaction. The solid line corresponds to the Glauber-Mueller dipole cross section whereas the dot-dashed one is the non-corrected DGLAP calculation.

As final comments, we address additional advantages of the color dipole picture in the DY case. For example, it allows to obtain the transverse momenta $p_{T}$ distribution for the process already at leading order calculation [17]. Instead, in the parton model the lepton pair has no transverse momentum due to the assumption that in the partonic subprocess the longitudinal momenta are bigger than the transverse ones (partons are collinear). Therefore, an alternative way to solve this trouble is to introduce an intrinsic $p_{T}$ for the initial state interacting partons. However, such an assumption is not sufficient to describe the measured $p_{T}$ distributions. Considering the Compton and the annihilation subprocess the leptons acquire transverse momentum and the $p_{T}$-dependence can be calculated in $\mathrm{pQCD}$. The resummations produce $\alpha_{s} \ln ^{2}\left(m^{2} / p_{T}^{2}\right)$ terms which are large as the transverse momentum goes to soft values $p_{T} \rightarrow 0$ (the perturbative expansion breaks down). Thus, the color 
dipole description is a nice tool to calculate that distributions since the above difficulties are avoided. One intends to address carefully this issue in a next calculation.

\section{CONCLUSIONS}

The Drell-Yan is an important process testing the quark (antiquark) content of the hadron target. The measured observables are Lorentz invariant, whereas the parton description is frame dependent. Calculations in the fast proton system have provided a perturbative understanding of DY up to higher orders. On the other hand, the color dipole picture allows a simple description of DY driven by the gluonic content (sea quarks) of the target. The quark from the projectile radiates a photon decaying into a lepton pair. The basic blocks in the color dipole are the LC wavefunctions and the dipole cross section. The former is calculated from perturbation theory and the latter one is modeled taking into account general properties of both hard and soft domains.

We have found that the LC wavefunction for the $\gamma^{*} q$ configuration plays the role of a weight function for the different transverse separations $r_{\perp}$ (as well as dipole sizes $\alpha r_{\perp}$ ) contribution to the process. Namely, small transverse separations are selected by both the transverse and longitudinal pieces. However, the transverse contribution can select nonnegligible large sizes $r_{\perp}$. In addition, larger invariant mass $M$ scans smaller $\gamma^{*} q$ separations. Moreover, the longitudinal piece is higher twist (suppressed by a factor $1 / M^{2}$ ). These features are also present in the deep inelastic case due to the similarity between the LC wavefunctions expressions.

Concerning the dipole cross section, here we consider the Glauber-Mueller approach, which takes into account the corrections to the standard DGLAP formalism due to the unitarity requirements. The taming of the parton distributions (parton saturation) at high energies is performed considering the multi-scattering assumption from the Glauber-like (eikonal) formalism. We have found a distinct behavior at both low and large $x_{2}$ when 
performing a comparison with the phenomenological GBW model. The main source of the deviation is that GM depends on the gluon distribution, which increases as $x_{2}$ diminishes. These features produce distinct results at current energies and in the forthcoming measurements. An important verification is that a non-negligible amount of non-perturbative contribution is present in the cross section. Although the LC wavefunctions suppress large transverse separations, a large cross section at small $x_{2}$ compensates the suppression producing significant soft content.

The current low $x_{2}$ available data lie in values ranging from $0.03 \leq x_{2} \leq 0.09$, actually testing the validity limit of the color dipole picture. Our results considering only GM dipole cross section underestimate the experimental measurements since color dipole includes only the sea quark content (gluon radiation) from the target. In the realistic case, for this kinematical region the valence and sea quarks have both a significant contribution in the cross section. We have parametrized the valence content through a reggeon exchange and the results turn out equivalent to the data description claimed in Ref. [17]. It was found also that issues related to higher orders contributions in the color dipole picture should be taken carefully.

As the energy reached in the forthcoming experiments increases, the saturation effects should turn out to be more relevant. We perform estimates for the RHIC energies and have found that the unitarity corrections are important in the description of the cross section. We expect that such correction should be larger at LHC, since the $x_{2}$ values probed there would be smaller than in RHIC.

The quite simple scenario for DY process in the rest frame allows to extend the approach to the nuclear case and also get information on $p_{T}$ distribution. The higher energies soon available will demand a well established knowledge on the nuclear gluon distribution which can be the input for the nuclear dipole cross section in the color dipole framework. This approach should be a useful tool to perform pQCD estimates for the future experimental measurements. 


\section{ACKNOWLEDGEMENTS}

M.A.B. and M.V.T.M. acknowledge useful discussions with Victor Gonçalves. M.V.T.M. also acknowledges Martin McDermott (Liverpool University-UK) for useful enlightenments. This work was supported by CNPq, BRAZIL. 


\section{REFERENCES}

[1] M. Klein, Int. J. Mod. Phys. A15S1, 467 (2000).

A.M. Cooper-Sarkar, R.C.E. Devenish and A. De Roeck, Int. J. Mod. Phys A13, 3385 (1998).

[2] Yu.L. Dokshitzer. Sov. Phys. JETP 46, 641 (1977);

G. Altarelli and G. Parisi. Nucl. Phys. B126, 298 (1977);

V.N. Gribov and L.N. Lipatov. Sov. J. Nucl. Phys 28, 822 (1978).

[3] A.H. Mueller, Nucl. Phys. 335, 115 (1990).

[4] A.L. Ayala, M.B. Gay Ducati, E.M. Levin, Nucl. Phys. B493, 305 (1997); Nucl. Phys. B511, 355 (1998).

M.B. Gay Ducati, V.P. Gonçalves, Nucl. Phys. B557, 296 (1999); Nucl. Phys. (Proc. Suppl.) 79, 302 (1999).

[5] A.L. Ayala, M.B. Gay Ducati, E.M. Levin, Eur. Phys. J C8, 115 (1999).

A.L. Ayala, M.B. Gay Ducati, V.P. Gonçalves, Phys. Rev. D59, 054010 (1999).

M.B. Gay Ducati, V.P. Gonçalves, Phys. Rev. C60, 058201 (1999); Phys. Lett. B466, 375 (1999).

[6] L.V. Gribov, E. M. Levin, M.G. Ryskin, Phys. Rep. 100, 1 (1983);

A.H. Mueller, Nucl. Phys. B558, 285 (1999); A.H. Mueller, hep-ph/0111244.

Y. U. Kovchegov, Phys Rev. D60, 034008 (1999).

I. Balitsky, Nucl. Phys. B463, 99 (1996).

L. Mc Lerran and R. Venugopalan, Phys. Rev. D49, 2233, 3352 (1994); 50, 2225 (1994);

53, $458(1996)$;

E. Iancu, A. Leonidov and L. Mc Lerran, Phys. Lett. B510, 133 (2001); Nucl. Phys. A 692, $583(2001)$;

E. Ferreiro, E. Iancu, A. Leonidov and L. McLerran, [hep-ph/0109115. 
J. Jalilian-Marian et al. Phys. Rev. D59, 034007 (1999);

N. Armesto and M. A. Braun, Eur. Phys. J. C20, 517 (2001).

[7] P.L. McGaughey, J.M. Moss, J.C. Peng, Ann. Rev. Nucl. Part. Sci. 49, 217 (1999).

[8] J.P. Blaizot, Theory of the Quark-Gluon Plasma, hep-ph/0107131.

[9] N. Arnesto, A. Capella, E.G. Ferreiro, Phys. Rev. C59, 395 (1999).

[10] N.N. Nikolaev, B.G. Zakharov, Z. Phys. C49, 607 (1991); Phys. Lett. B260, 414 (1991); Z. Phys. C53, 331 (1992).

[11] B.Z. Kopeliovich, In proceedings Workshop Hirschegg'95: Dynamical Properties of Hadrons in Nuclear Matter.. Ed. by H. Feldmeier and W. Nörenberg, Darmstadt, p. $102(1995)$.

S.J. Brodsky, A. Hebecker, E. Quack, Phys. Rev. D55, 2584 (1997).

B.Z. Kopeliovich, Phys. Lett. B447, 308 (1999).

[12] S.D. Drell, T.M. Yan, Phys. Rev. Lett. 25, 316 (1970).

[13] A. Vogt, Phys. Lett. B497, 228 (2001).

P.J. Rijken, W.L. van Neerven, Phys. Rev. D51, 44 (1995).

[14] G.R. Kerley, M. McDermott, J. Phys. G26, 683 (2000).

M.F. McDermott, Los Alamos preprint hep-ph/0008260.

[15] K. Golec-Biernat, M. Wüsthoff. Phys. Rev. D59, 014017 (1999); Phys. Rev. D60, 114023 (1999).

[16] M. Gluck, E. Reya, A. Vogt, Eur. Phys. J. C5, 461 (1998).

A. D. Martin et al. Eur. Phys. J. C(to appear) (2002), [hep-ph/0110215.

H, L, Lai et al. Eur. Phys. J. C12, 375 (2000).

[17] B.Z. Kopeliovich, J. Raufeisen, A.V. Tarasov, Phys. Lett. B503, 91 (2001). 
[18] E772 Collaboration, P.L. McGaughey et al., Phys. Rev. D50, 3038 (1994); erratum Phys. Rev. D60, 119903 (1999).

[19] M. McDermott et al., Eur. Phys. J. C16, 641 (2000).

L. Frankfurt, M. McDermott, M. Strikman, JHEP 0103, 045 (2001).

[20] E.A. Kuraev, L.N. Lipatov and V.S. Fadin. Phys. Lett B60 50 (1975); Sov. Phys. JETP 44443 (1976); Sov. Phys. JETP 45199 (1977);

Ya. Balitsky and L.N. Lipatov. Sov. J. Nucl. Phys. 28822 (1978).

[21] S. Catani, M. Ciafaloni, F. Hautmann, Nucl. Phys. B366, 135 (1991).

J.C. Collins, R.K. Ellis, Nucl. Phys. B360, 3 (1991).

[22] A. Donnachie, P.V. Landshoff, Phys. Lett. B437, 408 (1998).

[23] M.B. Gay Ducati. Braz. J. Phys. 31, 115 (2001) hep-ph/0107116.

[24] A. Kovner, U. A. Wiedemann, Phys. Rev. D64, 114002 (2001).

[25] A.L. Ayala, M.B. Gay Ducati, E.M. Levin, Phys. Lett. B388, 188 (1996).

M.B. Gay Ducati, V.P. Gonçalves, Phys. Lett. B502, 92 (2001).

[26] M.B. Gay Ducati, M.V.T. Machado, Phys. Rev. D (to appear), hep-ph/0111093.

[27] M. Gluck, E. Reya, A. Vogt, Z. Phys. C67, 433 (1995).

[28] M.B. Gay Ducati, V.P. Gonçalves, Phys. Lett. B487, 110 (2000).

[29] Huang Z, Lu HJ, Sarcevic I, Nucl. Phys. A637, 79 (1998).

[30] Gotsman et al., J. Phys. G27, 2297 (2001).

[31] H. Abramowicz, E. Levin, A. Levy, U Maor, Phys. Lett. B269, 465 (1991).

H. Abramowicz, A. Levy, hep-ph/9712415.

[32] M.G. Ryskin, A.G. Shuvaev, hep-ph/0203130] and references therein. 
[33] R. Kirschner, L.N. Lipatov, Nucl. Phys. B213, 122 (1983);

R. Kirschner, Z. Phys. C67, 459 (1995).

[34] A. Donnachie, P.V. Landshoff, Phys. Lett. B518, 63 (2001).

[35] B.Z. Kopeliovich, Phys. Lett. B447, 308 (1999).

[36] A. Bialas, H. Navelet, R. Peschanski, Nucl. Phys. B593, 438 (2001); Nucl. Phys. B603, $218(2001)$.

[37] G. Altarelli, R.D. Ball, S. Forte, Nucl. Phys. B599, 383 (2001). 